\title{
Optimization of a 3D micro-accelerometer based on diamagnetic levitation
}

\author{
Ch. Pigot, B. Delinchant, G. Reyne \\ G2ELab : Grenoble Electrical Engineering Lab \\ ENSIEG - BP 46 - 38402 Saint-Martin-d'Hères FRANCE \\ E-mail: christian.pigot@G2ELab.inpg.fr
}

\begin{abstract}
At the micro-scale, diamagnetic effects are of the same order of magnitude or even stronger than weight. Therefore, micro-magnets can levitate above diamagnetic bodies and move freely in the three dimensions of space. The possibilities and limits of a static and permanent levitating magnet are illustrated for the example of a 3-dimensional micro-accelerometer. Optimization is performed in order to fit most current market. It is shown that this technology fulfils the requirements for medium range and short time response markets.
\end{abstract}

Keywords: Diamagnetic levitation, micro-accelerometer, market specifications, micro-magnet, semi-analytical modelling, optimization

\section{Introduction}

Diamagnetism is the magnetic property of most of the everyday life materials. Usually it has a negligible effect. Nevertheless, it has been shown that scale reduction improves the volume diamagnetic forces allowing levitation of water or bismuth in air with simple micro-magnets [1]. The reverse effect (action-reaction) allows micro-magnets to levitate above diamagnetic material [2]. Thus passive, static, permanent and energy free levitation of a micro-magnet is possible at the micrometric scale.

However, there is not yet current use of the opportunities offered by such a technology. It may present huge advantages in the field of sensors, especially for inertial sensors. The example of a 3D accelerometer is given. Optimizations calculations are done in order to fulfil the specifications of most current accelerometers. These computations give an opportunity to explore the limits and interests of this technology. So through the validation of the commercial interest of this technology, the present work aims to explore the possibilities and the restrictions of diamagnetic levitation of a micro-magnet.

\section{Modelling}

An accelerometer is a sensor measuring an inertial force by a displacement of a proof mass. The proof mass here is a NdFeB micro-magnet levitating inside a diamagnetic box made of bismuth (Fig 1 left). Since it has no mechanical handling, it moves freely in the three dimensions of space, allowing independent measurements in all directions. Furthermore as the magnet approaches the diamagnetic material, it experiences a centering force pulling it back to the centre of the system. This force is linear for a certain distance and then grows exponentially (Fig 1 right). Thus this system is perfectly suited for use as a 3D micro-accelerometer. The linear part is used for measurement, while the exponential part allows the sensor to support high accelerations without damage.

Assuming that the diamagnetic material negligibly modifies the magnetic field, this field is analytically computed. This assumption is fully justified by the very weak susceptibility involved - around $10^{-5}$ and $10^{-4}$ for most materials. The force experienced by the magnet is then numerically calculated by surface integrals:

$$
F_{\text {dia / Magnet }}=-F_{\text {Magnet/dia }}=-\oiint_{\text {Sext }} \frac{\chi}{2 \mu_{0}} B^{2} d s \cdot \vec{n}
$$

with $\mu_{0}$ the permeability of the vacuum, $B$ the inducing field radiated by the magnet, Sext the surface of the diamagnetic shell and $\vec{n}$ the unitary normal outgoing vector. In our study, the magnet is polarized along the $\mathrm{Z}$ axis. The problem is then supposed to be identical along the $\mathrm{X}$ and $\mathrm{Y}$ axis. The modelling was performed in software describing algebraic equations of interactions between magnets and diamagnetic bodies [3].
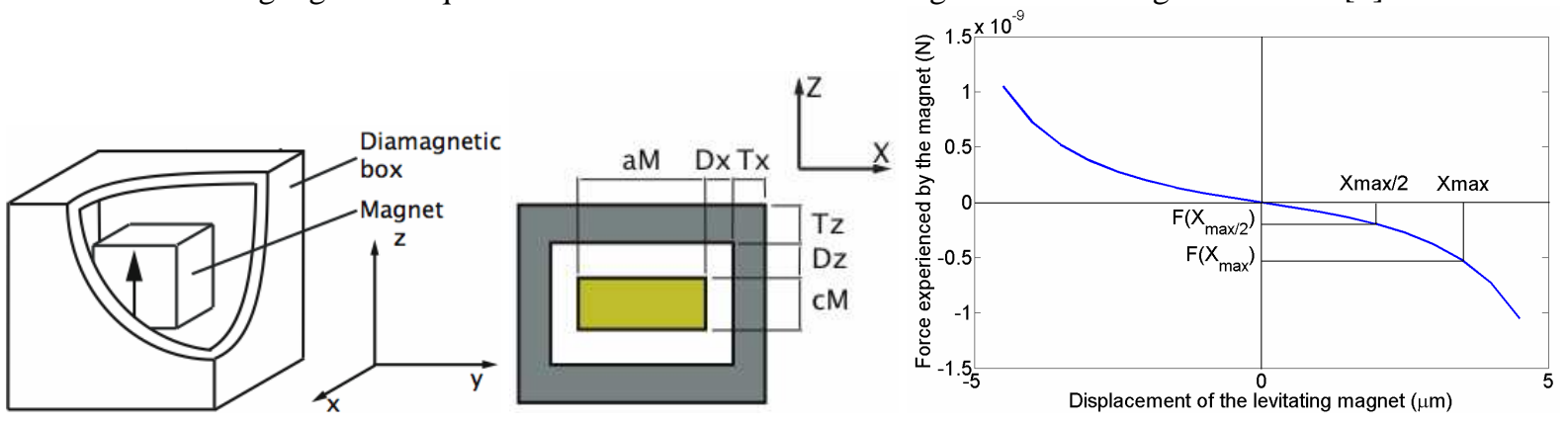

Fig.1: Modelling of a 3D accelerometer with a levitating $\mu$ magnet as proof mass,

Left: Isometric view, Center: Geometric parameters, Right: Force experience by the $\mu$ magnet along the $X$ axis 


\section{Optimization}

To fit the demand for current accelerometer markets, navigation, security and consumer applications are considered. A navigation application refers to positioning control to be used in correlation with a GPS; measurement precision is, then, the key parameter. A security application for use in automotive applications requires a fast time response. Lastly, for the consumer market aiming at human/machine interfaces, the price is decisive in the choice of the sensor. The specifications are summed up in Table 1.

The acceleration experienced by the system will be measured through the displacement of the magnet in the diamagnetic box. Thus the larger the displacement is, the easier and the more accurate the measurement will be. Therefore, the optimization aims to improve the displacement of the levitating body, with respect to the given specifications. Once the material is chosen, the physical and magnetic constants are fixed. Thus only the geometric dimensions of the system need to be optimized.

The optimizations were done in the CADES framework [4] where sensitivities of four constrained specifications to the geometric parameters were automatically computed from algebraic equations of the model. A sequential quadratic programming (SQP) algorithm was used in order to find the best solution for each of the three applications. One optimization with a standard PC takes about 10 iterations and less than 1 minute per iteration.

TABLE 1: REQUIERED SPECIFICATIONS

\begin{tabular}{|r|c|c|c|c|}
\hline Specifications & Definition & Consumer & Security & Navigation \\
\hline Linearity $(\%)$ & $\frac{F_{x \max }-2 \cdot F_{x \max / 2}}{F_{x \max }} \cdot 100$ & $<5$ & $<2$ & $<0,1$ \\
\hline Range $(\mathrm{g})$ & $\frac{F_{x \max }}{W}$ & $> \pm 2$ & $> \pm 2$ & $> \pm 1$ \\
\hline Resolution $(\mu \mathrm{g} / \sqrt{\mathrm{Hz}})$ & $\frac{\sqrt{4 \cdot k_{B} T \cdot f_{c}}}{M}$ & $<1000$ & $<1000$ & $<4$ \\
\hline Bandwidth $(\mathrm{Hz})$ & $\frac{F_{r e s}}{2}$ & $>20$ & $>400$ & $>100$ \\
\hline
\end{tabular}

With $\mathbf{F}_{\mathbf{c m a x}}$ and $\mathbf{F}_{\mathbf{x m a x} / 2}$ defined on Fig. 1, $\mathbf{W}$ the weight of the magnet, $\mathbf{M}$ its mass, $\mathbf{f}_{\mathbf{c}}$ the friction coefficient and $\mathbf{F}_{\mathbf{r e s}}$ the resonant frequency. Resolution depends on thermal activity defined by Boltzman constant, $\mathbf{k}_{\mathbf{B}}$, and the temperature $\mathbf{T}$

\section{Conclusions}

The performances along the most constrained axis (X or $\mathrm{Y}$ ) are summed up in Table 2. To respect the technology limits, geometrical parameters are bound within limits. Required range and bandwidth are reached without difficulties; nevertheless care has to be taken on linearity and resolution. In a nutshell, this technology offers new solutions for the consumer and security market requirements. However, for the navigation optimization the limits have been reached, thus specifications could not be fulfilled. Therefore applications requiring high accuracy need another technology.

Furthermore, the displacement range of the levitating body is known and maximized for both consumer and security applications. An appropriate non contact position measurement can now be chosen.

\section{TABLE 2: OPTIMIZATION RESULTS}

\begin{tabular}{|r|c|c|c|c|c|c|}
\hline Performance along the X axis & \multicolumn{2}{|c|}{ Consumer } & \multicolumn{2}{c|}{ Security } & \multicolumn{2}{c|}{ Navigation } \\
\hline Linearity $(\%)$ & 5,0 & $\checkmark$ & 2,0 & $\checkmark$ & 1,5 & $\mathbf{X}$ \\
\hline Range $(\mathrm{g})$ & 2,0 & $\checkmark$ & 2,0 & $\checkmark$ & 1,4 & $\checkmark$ \\
\hline Resolution $(\mu \mathrm{g} / \sqrt{\mathrm{Hz}})$ & 62 & $\checkmark$ & 200 & $\checkmark$ & 42 & $\times$ \\
\hline Bandwidth $(\mathrm{Hz})$ & 245 & $\checkmark$ & 400 & $\checkmark$ & 340 & $\checkmark$ \\
\hline Body displacement, $\mathrm{X}_{\max }(\mu \mathrm{m})$ & \multicolumn{7}{|c|}{2,0} & \multicolumn{7}{|c|}{0,76} & & \\
\hline
\end{tabular}

\section{REFERENCES}

[1] H. Chetouani,.V. Haguet, C. Jeandey, C. Pigot, A. Walther, N.M. Dempsey, F. Chatelain, B. Delinchant, G. Reyne, "Diamagnetic Levitation of Beads and Cells Above Permanent Magnets", TRANSDUCERS 2007, 715-718, 2007

[2] H. Profijt, C. Pigot, O. Cugat, G. Reyne, R. Grechishkin, "Diamagnetic levitation of a micro magnet by improvement of the magnetic gradients", J. Mag. Mag. Mat, submitted.

[3] H. Chetouani, B. Delinchant, G. Reyne, "Efficient modeling approach for optimization of a system based on passive diamagnetic levitation as a platform for bio-medical applications", COMPEL, Vol 26, Issue: 2, 2007

[4] B. Delinchant, D. Duret, L. Estrabaut, L. Gerbaud, H. Nguyen Huu, B. Du Peloux, H.L. Rakotoarison, F. Verdiere, F. Wurtz, “An optimizer using the software component paradigm for the optimization of engineering systems”, COMPEL Vol 26, Issue: 2, 2007 